REGARDS

SUR L'ECONOMIE ALLEMANDE

BULLETIN ECONOMIQUE DU CIRAC
Regards sur l'économie allemande

Bulletin économique du CIRAC

$73 \mid 2005$

Varia

\title{
A défaut d'alternance, une réelle perspective de changement
}

René Lasserre

\section{OpenEdition}

1 Journals

Édition électronique

URL : http://journals.openedition.org/rea/640

DOI : $10.4000 /$ rea. 640

ISBN : 978-2-8218-0842-3

ISSN : 1965-0787

Éditeur

CIRAC

Édition imprimée

Date de publication : 1 octobre 2005

Pagination : 1-2

ISSN : 1156-8992

Référence électronique

René Lasserre, «A défaut d'alternance, une réelle perspective de changement », Regards sur l'économie allemande [En ligne], 73 | octobre 2005, mis en ligne le 30 avril 2008, consulté le 22 septembre 2020

URL : http://journals.openedition.org/rea/640 ; DOI : https://doi.org/10.4000/rea.640 


\section{A défaut d'alternance, une réelle perspective de changement}

Le résultat des élections fédérales du 18 septembre a été pour le moins inattendu : le Chancelier Schröder n’a pas réussi à obtenir la majorité renforcée qu'il espérait en provoquant la dissolution du Bundestag. Mais alors qu'il était parti largement perdant, il a effectué une spectaculaire remontée dans l'opinion et échoué de peu à faire gagner son parti sur le fil. L'échec d'Angela Merkel n'en est que plus patent : en refusant finalement de donner la majorité à une coalition nouvelle, les électeurs ont clairement rejeté l'alternative néo-libérale qu'elle entendait promouvoir.

L'alternance politique tant annoncée n'aura donc pas lieu, et à défaut d'autre solution politiquement viable et crédible, l'on s'achemine vers une "grande coalition ", réunissant les deux principaux partis autour d'un programme commun de gouvernement. Cette perspective, surprenante à première vue, ne constitue pas pour autant un pis-aller : même si elle se heurte à de multiples obstacles et présente de réels dangers, elle apparaît en fin de compte comme la voie la plus sûre qui mène au changement.

La constitution d'une grande coalition a d'abord pour elle la garantie incontestable de la légitimité, car elle répond à la volonté perplexe des électeurs. En ne donnant de majorité à aucune des deux coalitions en présence, tout en sanctionnant les deux grands partis, les citoyens ont en fait appelé ces derniers à dépasser leurs clivages et à trouver ensemble, sur la base d'une large majorité de gouvernement, les réponses politiques aux problèmes qu'ils n'ont pas su jusqu'à présent résoudre séparément. Dans son issue apparemment contradictoire, le vote est en réalité un appel aux deux grands partis à exercer conjointement leurs responsabilités pour opérer les choix qui s'imposent et sortir le pays du marasme.

Il serait en effet erroné d'interpréter le vote du 18 septembre à travers le seul prisme de l'immobilisme et du refus du changement. Cela ne vaut tout au plus que pour les quelque $12 \%$ d'électeurs qui, d'ailleurs plus à gauche qu'à droite, se sont portés aux extrêmes de l'échiquier politique. Il s'agit au contraire d'un mandat politique explicite en faveur des réformes, non plus seulement sur leur principe, désormais partagé par les deux grands partis, mais bien sur l'obligation de créer enfin les conditions politiques de leur mise en œuvre. Ce mandat politique place les deux partis majoritaires au pied du mur, car il comporte non seulement une obligation de moyens, mais aussi de résultats.

Tout d'abord, une obligation de moyens, avec la nécessité de faire preuve d'une réelle volonté d'aboutir et de surmonter rapidement les préalables politiques, à commencer par les questions de personnes, que ce soit pour la désignation du Chancelier ou pour la répartition des responsabilités au sein du futur gouvernement, laquelle reflétera ses équilibres politiques, tout en étant le gage de sa cohésion et de sa crédibilité.

Une obligation de résultats ensuite, avec la nécessité de parvenir, à échéance raisonnable, à un programme solide de gouvernement. Car à défaut d'avoir présenté chacun de leur côté des propositions convaincantes, les deux partis devront rapidement afficher des objectifs clairs 
et partagés, qui ne recueilleront le soutien d'une large frange de l'opinion que pour autant qu'ils parviendront à changer la donne sur quelques grands dossiers. L'exercice est tout à fait possible et d'ailleurs déjà largement balisé, puisqu'à travers le fédéralisme coopératif entre Bund et Länder, SPD et CDU ont déjà de longue date pratiqué le compromis et assumé des politiques conjointes, que ce soit dans le domaine fiscal, ou dans ceux de la protection sociale ou du marché du travail. Dans le cadre d'une responsabilité politique désormais partagée au niveau fédéral, cet acquis constitue un gage d'efficacité incontestable. Par ailleurs, l'alliance entre les deux partis permettra de part et d'autre de mobiliser plus étroitement encore les partenaires sociaux sur des objectifs prioritaires d'intérêt général. Le précédent de 1966-69 avait permis de donner un cadre d'action politique nouveau qui a fait ses preuves dans la durée.

Reste que dans le contexte économique actuel, l'exercice sera singulièrement plus délicat car il impliquera avant tout des sacrifices, et peu de contreparties. Autant les lignes d'entente pourront être définies sans obstacles insurmontables dans le domaine fiscal et budgétaire, et sans doute également sur la question cruciale de la réforme du fédéralisme, autant le point d'équilibre sera difficile à trouver dans le domaine social, en particulier dans le mode de financement des régimes sociaux qui conditionne à la fois le niveau des coûts salariaux et celui de la couverture sociale. Les arbitrages seront rudes car les sociaux-démocrates ne manqueront pas de se prévaloir des leçons du scrutin du 18 septembre pour veiller à préserver un socle d'acquis sociaux fondamentaux. La question de la flexibilisation des conditions de rémunération et d'emploi constitue aussi un terrain sensible, sur lequel la négociation pourrait achopper. Encore s'agit-il de quelques ajustements sur lesquels des transactions sont possibles, tant sur ce dossier beaucoup d'avancées ont déjà été réalisées avec la réforme Hartz IV, tandis que les partenaires sociaux ont parcouru au cours de la décennie écoulée un chemin considérable en matière de flexibilité négociée.

La perspective d'une grande coalition devrait ainsi pouvoir se concrétiser autour de quelques objectifs significatifs en matière économique, en particulier avec la mise en œuvre d'une politique de consolidation budgétaire et financière et de restructuration des prélèvements fiscaux et sociaux. Les objectifs devront être d'autant plus clairement affichés qu'il s'agit de politiques de longue haleine et qu'il importe avant tout de restaurer la confiance et d'assurer aux acteurs économiques et sociaux une visibilité à moyen terme.

Mais c'est peut-être dans sa dimension politique qu'une grande coalition offre à court terme une réelle perspective de renouveau : la recherche de majorités larges et solidaires sur quelques dossiers essentiels devrait permettre à la classe politique de surmonter les blocages institutionnels qui depuis plus d'une décennie entretiennent l'immobilisme et conduisent à la paralysie politique. La chance est offerte aux deux grands partis de redonner du sens à l'action réformatrice et de refonder ainsi le pacte démocratique et social. Ils ne peuvent ni ne doivent la laisser échapper.

René Lasserre (07-10-05) 